\title{
Incidencia de los procesos educomunicativos en los proyectos ambientales escolares*
}

\author{
Ángela María Bedoya Mejía**, Luz Bibiana Moscoso Marín ${ }^{\star * *}$, Lina María Rendón López ${ }^{* \star *}$
}

\section{Resumen}

Introducción. Se presenta un análisis cualitativo y cuantitativo de la incidencia de los procesos educomunicativos en la educación, específicamente en los Proyectos Ambientales Escolares de la Red PRAE del Área Metropolitana del Valle de Aburrá, a través de reflexiones críticas acerca de la importancia de la contextualización de la educación ambiental y la manera más eficaz de transmitir sus procesos y resultados a toda la comunidad educativa. Materiales y métodos. Se planteó el diseño y aplicación de instrumentos de recolección de información primaria con algunos miembros de la comunidad educativa de cada institución intervenida a través de la realización de encuestas y entrevistas a profundidad. Se almacenó la información en bases de datos y se analizó a través de métodos gráficos y valores porcentuales, obteniendo las tendencias en la información recolectada. Resultados. Se encontró que los procesos de comunicación interna en las instituciones educativas son muy débiles, lo que redunda en unos niveles bajos de apropiación de los proyectos. La mayoría de las instituciones educativas ha iniciado procesos de formación y capacitación para los diferentes actores pero con pocos resultados, debido a que la información que se brinda no es multiplicada a la totalidad de la comunidad educativa. Conclusión. Hace falta mayor divulgación de resultados que hagan más visibles los propósitos y logros de los proyectos ambientales escolares, e ir más allá de la formulación de proyectos para hacer una lectura más amplia del contexto, identificando las verdaderas potencialidades del mismo a través de la solución de los problemas reales que los aquejan.
Palabras clave: educación ambiental, educomunicación, PRAE.

\section{Influence of educommunicative processes on environmental projects at schools}

\begin{abstract}
Introduction. A qualitative and quantitative analysis of the influence of educommunicative processes on education, specifically on the School Environmental Projects of the PRAE Network at Área Metropolitana del Valle de Aburrá, is introduced in this article. This is done by means of critical reflections about the importance of contextualization of the environmental education and the most effective way to transmit their processes and results to the whole educative community. Methodology. The design and application of the collection instruments for the primary information were proposed with some members of the educative community from every institution in which the project was applied, by means of surveys and in-depth interviews. The information was stored in databases and analyzed by the use of graphic methods and percentages, thus obtaining the tendencies in the information collected. Results. The internal communication processes in the educative institutions are very weak, so the appropriation of the projects has not been enough. Most of the institutions have started training processes for the actors involved, but have had poor results due to the fact that the formation provided is not fully multiplied for the whole educative community. Conclusion. More divulgation of the results is necessary in order to make the purposes and achievements of the
\end{abstract}

\footnotetext{
Artículo original derivado de la investigación "Fortalecimiento de los PRAE apoyado en procesos educomunicativos" en el marco del convenio Red PRAE N. ${ }^{\circ} 962$ de 2013. Área Metropolitana del Valle de Aburrá, CORANTIOQUIA y Corporación Universitaria Lasallista.

** Magíster en Planificación de Proyectos de Desarrollo Rural y Gestión. Docente Programa de Ingeniería Ambiental. Corporación Universitaria Lasallista.

*** Magíster en Desarrollo Sostenible y Medio Ambiente. Docente Programa de Ingeniería Ambiental.

**** Magíster en Comunicaciones. Docente de la Corporación Universitaria Lasallista.
}

Autor para correspondencia: Ángela María Bedoya Mejía, email: anbedoya@lasallistadocentes.edu.co Artículo recibido: 14/082015; Artículo aprobado: 18/08/2015. 
environmental projects at the schools more visible, going beyond the formulation of the projects and making a wider reading of the context, identifying the real strengths of this context through the solution of the real problems these projects are having.

Key words: environmental Education, Educommunication, PRAE

\section{Incidência dos processos educomunicativos nos projetos ambientais escolares Resumo}

\section{Resumo \\ Introdução. Apresenta-se uma análise qualitativa e quantitativa da incidência dos processos educomunicativos na educação, especificamente nos Projetos Ambientais Escolares da Rede PRAE da Área Metropolitana do Vale de Aburrá, através de reflexões críticas a respeito da importância da contextualização da educação ambiental e a maneira mais eficaz de transmitir seus processos e resultados a toda a comunidade educativa. Metodologia. Propôs-se o desenho e aplicação}

de instrumentos de recolha de informação primária com alguns membros da comunidade educativa de cada instituição intervinda através da realização de enquetes e entrevistas a profundidade. Armazenouse a informação em bases de dados e se analisou através de métodos gráficos e valores porcentuais, obtendo as tendências na informação coletada. Resultados. Se encontrou que os processos de comunicação interna nas instituições educativas são muito débeis, o que implica a uns níveis baixos de apropriação dos projetos. A maioria das instituições educativas iniciaram processos de formação e capacitação para os diferentes atores mas com poucos resultados, devido a que a informação que se brinda não é multiplicada a totalidade da comunidade educativa. Conclusão. Faz falta maior divulgação de resultados que façam mais visíveis os propósitos e conquista dos projetos ambientais escolares, ir além da formulação de projetos fazendo uma leitura mais ampla do contexto, identificando as verdadeiras potencialidades do mesmo através da solução de problemas reais aos quais estão submetidos.

Palavras chaves: Educação Ambiental, Educomunicação, PRAE

\section{Introducción}

La educación ambiental es una compleja dimensión de la educación global, caracterizada por una gran diversidad de teorías y de prácticas que abordan desde diferentes puntos de vista la concepción de educación, de medio ambiente, de desarrollo social y de educación ambiental (Sauvé, 2003).

En este sentido, la UNESCO señala que es fundamental contar con estrategias adecuadas de comunicación y educación para el desarrollo sostenible (CEDS) que nos ayuden a entender qué hacer, por qué lo hacemos, y cómo hacerlo mejor. Son las que nos permiten percibir qué pasaría si no mejoramos y qué áreas de nuestra vida se afectarían ante nuestra inacción (Badillo, 2011).

En Colombia, todas las reflexiones que se han hecho y se siguen haciendo en torno a la educación ambiental en el contexto se basan en la necesidad de contribuir a la formación de ciudadanos capaces de relacionarse en forma adecuada con el ambiente, teniendo en cuenta las necesidades actuales y las propuestas de desarrollo sostenible que se vienen construyendo en las diferentes regiones del país, con el ánimo de trascender logros aislados y momentáneos en materia de manejo de los recursos naturales y del entorno (Carrasco, 1998).

Entre los medios que se han venido utilizando en Colombia para propiciar espacios de reflexión se encuentran las conceptualizaciones y estrategias planteadas en foros y seminarios nacionales e internacionales, ya que formaban entonces un ambiente favorable para evaluar lo que estaba sucediendo en el campo de la educación ambiental en el país, dado que en esos eventos se venían discutiendo y delineando políticas globales muy importantes sobre el tema (Carrasco, 1998).

Los proyectos ambientales escolares (PRAE) se convierten, entonces, en una de las estrategias de la Política Nacional de Educación Ambiental (PNEA) en Colombia para articular la dimensión ambiental a los procesos de formación en el ámbito de la educación formal, 
desde las perspectivas de conceptualización, contextualización, sistematización y proyección de estos proyectos (Educación, 2002).

Los PRAE se articulan a la educación y esta, en todos sus momentos y modalidades es, en esencia, un proceso de comunicación, no solamente entre seres humanos que compartimos un mismo espacio (real o virtual) y unas mismas circunstancias de temporalidad, sino también de comunicación entre el presente y el pasado. La comunicación nos permite conectar el hoy con el ayer y entender lo que somos en función de procesos en marcha (Wilches-Chaux, 2006).

Por lo anterior el sistema educativo en Colombia viene implementando los PRAE en la educación formal. Sin embargo, en muchas ocasiones los procesos se han debilitado debido a las deficiencias comunicativas, tales como espacios de reflexión en torno a la educación ambiental, ejercicios investigativos y desarrollos del proyecto que no tienen estrategia de difusión óptima para que toda la comunidad educativa conozca y se apropie de dichos procesos.

Considerando la complejidad de la educación ambiental, en los PRAE cabe resaltar la importancia del "enfoque colaborativo y participativo" donde, según Sauvé (2003), el medio ambiente se convierte en un objeto esencialmente compartido que requiere ser abordado conjuntamente para hacer converger en él las miradas, las esperanzas y los talentos de cada uno. En ese sentido, la pedagogía de la educación ambiental estimula a los profesores a trabajar en equipo, incluso con los demás miembros de la comunidad educativa. De esta manera se puede aprender unos con otros y unos de otros. En esto podemos identificar los principios del socio-constructivismo como una forma de comunicación interna de gran validez para fortalecer los PRAE en el interior de la comunidad educativa.

Igualmente el diseño, la implementación, el apoyo y la promoción de planes y acciones de comunicación y divulgación son fundamentales, pues de ellos dependen el acceso ágil y oportuno a la información ambiental y la facilitación de instancias de diálogo entre la sociedad civil y el
Estado. Los procesos complejos de reflexión, investigación, concienciación y sensibilización en los que se enfoca la educación ambiental deberán estar basados en estrategias educomunicativas nutridas de sentidos educativos y pedagógicos.

Se pueden analizar dos formas de la comunicación que, de manera no siempre explícita, pero sí muy eficaz, constituyen también educación: una, nuestra relación con los medios masivos de comunicación, como el periódico, el cine, la radio, la televisión $y$, por supuesto, la Internet, con todas las posibilidades que este medio ofrece para una real interactividad; otra, los procesos de participación. Ambas formas de comunicación transforman al ser humano para bien o para mal. Unas veces refuerzan los aprendizajes que se adquieren en la educación formal, otras los contradicen, también para bien o para mal. En uno u otro proceso se construyen seres humanos y de alguna manera (generalmente pasiva en el contacto convencional con los medios de comunicación), también se construye comunidad (Wilches-Chaux, 2006).

\section{Materiales y métodos}

La investigación planteada utilizó una metodología combinada, pues hubo momentos en donde se hizo uso de una metodología cuantitativa y momentos en donde se aplicó la metodología cualitativa. Para alcanzar el objetivo propuesto en esta investigación, se desarrolló una propuesta metodológica, que se describe a continuación:

\section{Planteamiento del problema}

Para esta investigación se espera generar herramientas de información y comunicación vía online, ya que podrían facilitar la participación activa de las comunidades educativas. De acuerdo con Soares (2000), la fusión entre los campos de la educación y la comunicación ha creado uno nuevo, eminentemente dialógico, que si bien conserva muchas de las características de cada uno de ellos, también genera unos nuevos e idénticos movimientos. Es por esto que la investigación pretende dar solución a la siguiente pregunta 
orientadora: ¿Cómo incide la educomunicación en los proyectos ambientales escolares?

\section{Diseño y aplicación de herramientas}

Apoyados en la revisión bibliográfica y la reflexión teórica, se planteó el diseño y aplicación de instrumentos de recolección de información primaria con algunos miembros de la comunidad educativa de cada institución intervenida a través de la realización de encuestas y entrevistas a profundidad.

La encuesta se trata de uno de los instrumentos de uso más universal en la investigación; es un método basado en una serie de preguntas o ítems dirigidos a sujetos con el fin de describir y/o relacionar acerca de un determinado problema sobre el que se desea indagar (Del Rincón et al., 1995). Por su parte, las entrevistas permiten la recogida de información través de un proceso de comunicación directa en el que el entrevistado responde a cuestiones, previamente diseñadas por el entrevistador (Buendía, Colas y Hernández, 2010).

\section{Recolección de datos}

A través de las herramientas diseñadas, se estableció el método de recogida de información cualitativa y cuantitativa, de manera que los datos pudieran ser interpretados y analizados con el fin de dar respuesta al problema planteado.

Se eligieron entonces, en cada institución educativa, los representantes del PRAE de los niveles directivo, docente y estudiantil. Por cada institución se eligieron tres personas a las que se les aplicó la entrevista, basados en el guion previamente diseñado, tratando de mantener el curso de la discusión. Asimismo, se aplicaron las encuestas a tres grupos completos de estudiantes, preferencialmente, de los grados 6, 9 y 11 .

Toda esta información fue almacenada en bases de datos para su posterior análisis.

\section{Análisis e interpretación}

La base de datos generada con las encuestas comenzó a analizarse a través de métodos gráficos y valores porcentuales, extrayendo de esta manera, las tendencias en la información recolectada. Por su parte, las entrevistas permitieron identificar las percepciones de los diferentes actores de las instituciones educativas y así identificar las opiniones sobre los temas planteados, permitiendo evaluar el nivel de conocimiento de los mismos sobre los objetivos, alcances y niveles de desarrollo, logrados a través de los procesos educomunicativos.

\section{Informe}

Se elaboró un documento que contenía toda la información obtenida y analizada, y a partir de ella, se emitió una serie de conclusiones y recomendaciones, acerca del problema planteado y al cual se trató de dar respuesta con el desarrollo de la metodología. Se realizaron, además, encuentros con todas las instituciones educativas participantes, donde se expusieron sus logros y avances, se fortaleció el PRAE a través de una serie de iniciativas que cada Institución planteó integrando a todos los miembros de su comunidad $\mathrm{y}$, además, se diseñó y entregó una cartilla con los logros y avances alcanzados en la Red.

\section{Resultados y discusión}

Los procesos de comunicación interna en las instituciones educativas son muy débiles, lo que dificulta la apropiación de los proyectos, como es el caso del PRAE. En la mayoría de las instituciones educativas se han iniciado momentos de formación y capacitación para los diferentes actores del PRAE pero con pocos resultados, debido a que la información que se brinda se queda en unos pocos actores y no es multiplicada a totalidad de la comunidad educativa.

Los medios utilizados por las instituciones educativas para comunicar procesos como el PRAE se quedan en las circulares y en las carteleras, y dejan al receptor del mensaje la autonomía para una interpretación, en ocasiones errónea, de la información.

La encuesta se aplicó a 181 estudiantes de diferentes instituciones educativas que 
participaron del convenio 962 de 2013, RED PRAE Área Metropolitana del Valle de Aburra y se aplicaron preguntas como las que se muestran a continuación:

¿Conoces la existencia del proyecto ambiental escolar (PRAE) en tu institución educativa?

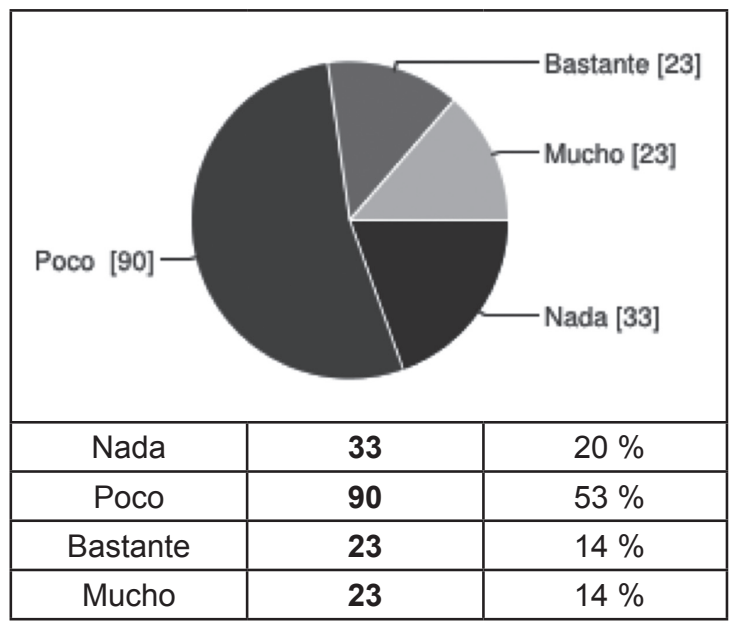

Más de 120 estudiantes manifiestan conocer nada o poco del PRAE y solo 46 manifiestan conocer bastante o mucho, lo que podría indicar que los sistemas de difusión del proyecto no han sido suficientemente efectivos.

¿Conoces si en tu institución educativa existe un proyecto ambiental?

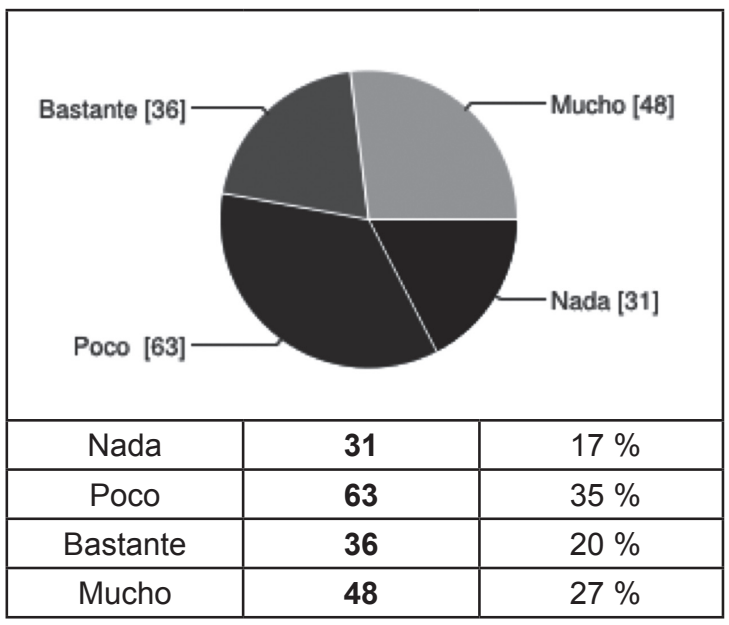

Existe un cierto equilibrio entre el número de estudiantes que manifiesta no conocer nada o poco el proyecto y aquellos (94 alumnos) que manifiestan conocerlo bastante o mucho.

¿En qué grado consideras que se han comunicado las actividades propias del PRAE, tales como formulación del proyecto, reuniones de planeación, jornadas de intercambio, lectura de contexto, entre otras?

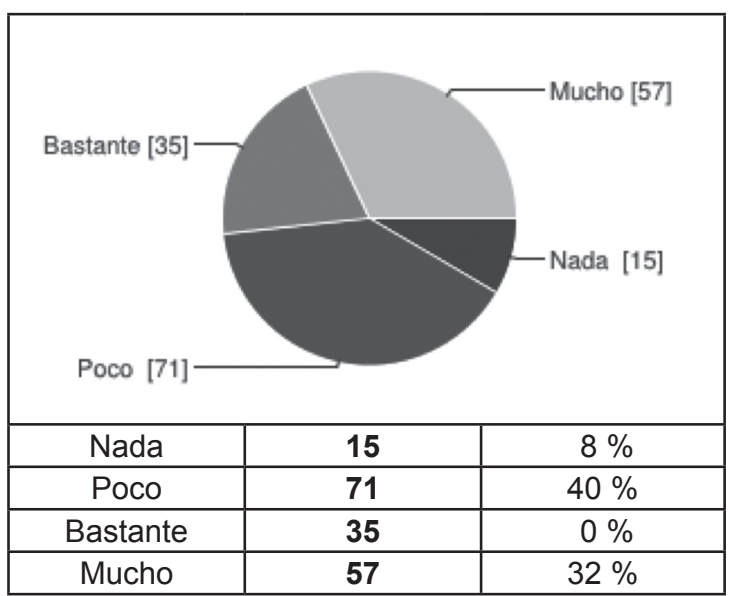

Cuando se indaga por el grado de comunicación de las actividades propias del PRAE, tales como formulación del proyecto, reuniones de planeación, jornadas de intercambio, lectura de contexto, prácticamente la mitad de quienes respondieron asegura que las actividades del PRAE se han comunicado nada o poco, frente a la otra mitad que asegura que se han comunicado bastante o mucho.

Selecciona cuál o cuáles de los siguientes medios han servido al PRAE para difundir sus actividades tales como formulación del proyecto, reuniones de planeación, jornadas de intercambio, lectura de contexto, entre otras, al interior de la institución educativa:

Existe una gran dispersión en las respuestas referidas a los medios de difusión de las actividades del PRAE; se destaca la información mediante carteles, seguida a cierta distancia de la información voz a voz, mediante afiches y mediante capacitaciones en talleres. 


\begin{tabular}{|c|c|c|c|c|c|}
\hline Periódico mural & 22 & $5 \%$ & Redes sociales & 11 & $3 \%$ \\
\hline Carteleras & 93 & $23 \%$ & Capacitaciones y talleres & 58 & $14 \%$ \\
\hline Afiches & 67 & $17 \%$ & Voz a voz & 62 & $15 \%$ \\
\hline Folletos & 15 & $4 \%$ & Grupos de discusión & 11 & $3 \%$ \\
\hline Circulares informativas & 37 & $9 \%$ & Página web & 15 & $4 \%$ \\
\hline Otro & \multicolumn{3}{|c|}{$15 \%$} \\
\hline
\end{tabular}

En caso de no haber comunicación de actividades tales como formulación del proyecto, reuniones de planeación, jornadas de intercambio, lectura de contexto, entre otras, en el interior de la Institución Educativa, selecciona la que consideres sea la razón o las razones por las que sucede esto (puedes seleccionar una o varias opciones):

\begin{tabular}{|l|c|c|}
\hline $\begin{array}{l}\text { No hay interés en la comunidad hacia } \\
\text { el proyecto }\end{array}$ & 48 & $26 \%$ \\
\hline Faltan los medios de divulgación & 40 & $22 \%$ \\
\hline $\begin{array}{l}\text { Las estrategias de divulgación son } \\
\text { poco efectivas }\end{array}$ & 50 & $27 \%$ \\
\hline No hay presupuesto para divulgación & 20 & $11 \%$ \\
\hline $\begin{array}{l}\text { El docente encargado del PRAE no } \\
\text { divulga }\end{array}$ & 13 & $7 \%$ \\
\hline $\begin{array}{l}\text { No hay acceso a los medios de } \\
\text { comunicación }\end{array}$ & 9 & $5 \%$ \\
\hline Otro & 5 & $3 \%$ \\
\hline
\end{tabular}

Entre las razones que los encuestados señalan para explicar la inexistencia de la comunicación de las actividades al público, destacan la poca efectividad de las estrategias de comunicación, la falta de medios y el posible desinterés por tal divulgación.

¿En qué grado consideras que se comunican los resultados obtenidos con la ejecución del PRAE?

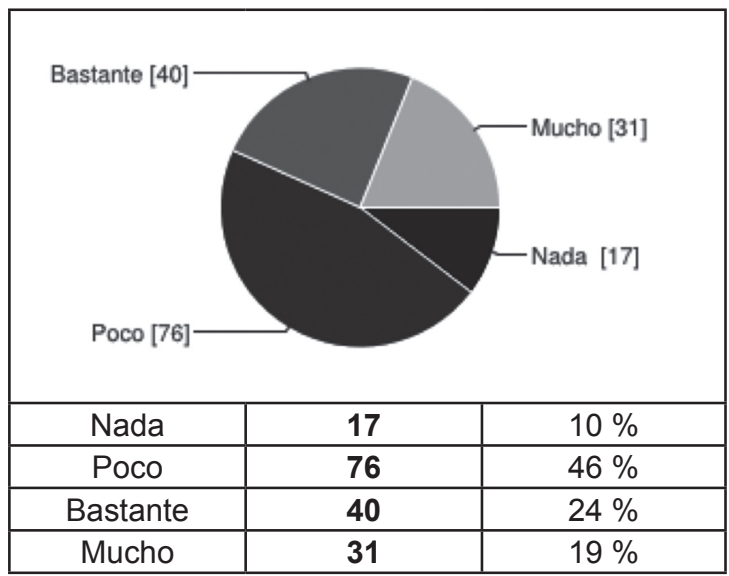

Casi un centenar de estudiantes encuestados respondieron que los resultados del PRAE se han difundido poco o nada, mientras que unos 70 opinan lo contrario, es decir, que los resultados se han difundido bastante o mucho.

Selecciona uno o varios de los medios que consideras, son eficaces para comunicar los elementos del PRAE y los resultados de su ejecución:

Los encuestados manifiestan que las principales razones por los que no hay una asertiva comunicación de resultados del PRAE se deben a la falta de interés en la comunidad educativa hacia el PRAE, a que faltan medios de divulgación y las estrategias existentes son poco efectivas.

A pesar de la cercanía que tienen los estudiantes con las tecnologías digitales, se observa que son los medios tradicionales los que consideran más eficaces para comunicar los elementos del PRAE y los resultados de su ejecución. Cabe anotar que existe una enorme variedad de opinión sobre los medios de comunicación eficaces, si bien se destaca la importancia que le dan al uso de carteleras y la poca importancia a los medios digitales.

En las entrevistas realizadas a directivos $y$ docentes de las instituciones educativas se evidenció que los procesos de comunicación virtual con la planta docente son difíciles de aplicar, puesto que no son muy activos en este tema y en muchas ocasiones ni la institución ni el docente cuenta con los medios ni la conectividad requerida; por ello la comunicación se reduce la mayoría del tiempo a estrategias de comunicación tradicional de tal manera que se garantice al máximo la transmisión de la información.

Esta situación afecta radicalmente la intención de dar a conocer el PRAE a toda la comunidad 
educativa. Los entrevistados manifestaron grandes debilidades en los procesos de comunicación interna, lo que interfiere en la consecución de las metas establecidas para conformar Red. Los mecanismos y procesos de comunicación deberían estar definidos y estructurados teniendo en cuenta la pertinencia según el contexto de cada institución educativa, con la finalidad de dar sostenibilidad a la Red PRAE.

\begin{tabular}{|c|c|c|c|c|c|}
\hline Página web & 50 & $8 \%$ & Periódico mural & 29 & $5 \%$ \\
\hline Redes sociales & 37 & $6 \%$ & Informe final escrito & 16 & $3 \%$ \\
\hline Blog & 34 & $6 \%$ & Talleres y encuentros & 57 & $9 \%$ \\
\hline Folletos & 56 & $9 \%$ & Emisora & 37 & $6 \%$ \\
\hline Circulares & 33 & $5 \%$ & Videos & 53 & $9 \%$ \\
\hline Carteleras & 84 & $14 \%$ & Fotografías & 54 & $9 \%$ \\
\hline Afiches & 61 & $10 \%$ & Otro & 8 & $1 \%$ \\
\hline
\end{tabular}

Sin embargo, existen algunas experiencias interesantes que cumplen objetivos de comunicación del PRAE a toda la comunidad educativa, no solo en el interior de la institución sino que se proyecta a toda la comunidad como lo señala uno de los docentes entrevistados:

\begin{abstract}
Al interior del PRAE utilizamos conversatorios con los docentes y con el grupo de estudiantes líderes, encuentros con padres de familia, jornadas pedagógicas con docentes, semanas ambientales, videos, socialización, canal local de televisión, cartelera especial. Durante las clases hacemos grabaciones y hacemos socializaciones de cómo les pareció el proyecto referente a la parte de proyectarnos a la comunidad tenemos un periódico que nos apoya que se llama Meridiano 75 que es local y él muestra todo lo que hemos realizado hasta la fecha y todos los proyectos que nosotros hacemos salen publicados allí, a ellos les pareció muy interesante. A parte de eso, tratamos de formar apoyo con la universidad San Buenaventura, con la Secretaria de Educación, con los trabajadores de Piamonte que son los que están en nuestro territorio, en nuestro contexto y podemos articular con ellos algunas ideas y dinámicas de experiencias.
\end{abstract}

En otra de las instituciones educativas se desarrolla una estrategia de comunicación que vincula el PRAE con otros proyectos pedagógicos como el proyecto eco-club, el proyecto de vida y los encuentros de padres de familia.
Asimismo como hay experiencias exitosas, también se observó a través de las entrevistas que muchos directivos están descontextualizados respecto a lo que realmente son los proyectos ambientales escolares y no dan importancia a la divulgación de los mismos.

La alta rotación de personal en las instituciones educativas es un factor que retrasa los procesos del PRAE, puesto que en repetidas ocasiones deben volver a empezar con los proyectos.

Los estudiantes que fueron entrevistados hacen parte del grupo dinamizador del PRAE y tienen en muchas ocasiones mayor claridad respecto a los lineamientos definidos por la Política Nacional de Educación; en cuanto al retroceso debido a la rotación de directivos y docentes, un estudiante manifestó: "el PRAE dejó de ser ambiental y pasó a ser ecológico, el activismo se volvió obligatorio, hubo un retroceso social y se volvió un proyecto de ciencias".

En este sentido, menciona Badillo (2011), en un acercamiento similar a los proyectos en el departamento del Valle del Cauca, gran parte de los PRAE están siendo acogidos por los directivos y docentes como proyectos formales obligatorios, y no como una oportunidad metodológica para una mejor formación de sus docentes, de sus estudiantes y de la comunidad educativa en general.

Adicionalmente, se considera fundamental avanzar en procesos centrados en el 
componente comunicativo y pedagógico, dirigidos al uso de las tecnologías de la información y la comunicación (TIC), pues si bien el diagnóstico mencionado da referencia al uso de herramientas de educación ambiental (impresos, audiovisuales), la revisión documental sobre la problemática indica que es necesario profundizar en las estrategias de comunicación y pedagogía empleadas (Badillo, 2011).

Es por esto, que tal y como menciona Aguaded (2012) la educomunicación debe concebirse como una herramienta necesaria, pues, la sociedad audiovisual reclama un contexto educativo en el que los medios han de jugar un papel crucial. Si nuestra escuela ha de preparar a los alumnos de hoy a vivir en la sociedad de forma autónoma y libre, no puede seguir ignorando los medios. No queda otra alternativa que integrarlos didácticamente y sacar de ellos todo lo positivo que puedan ofrecernos.

\section{Conclusiones}

Hoy en día, y a pesar del paso del tiempo y de la evolución de las tecnologías de la comunicación y la información, las carteleras y los afiches, siguen siendo en el interior de las instituciones los medios más usados para la divulgación de este tipo de proyectos, y quedan relegados a un rincón poco visitado de la Institución Educativa y con información bastante fragmentada acerca de la planeación, ejecución y obtención de logros al nivel de proyecto institucional, o la distorsión de la información en las comunicaciones "voz a voz", al punto que lo único que subsiste y persiste en el tiempo, según los datos, son informes escritos a los cuales poco acceso tienen muchos de los actores del proceso, tales como estudiantes, personal de apoyo en las instituciones (aseo, vigilancia, alimentos, entre otros) e inclusive la misma comunidad circundante a la Institución.

Hace falta mayor divulgación de resultados que hagan más visibles los propósitos y los logros de los proyectos ambientales escolares, ir más allá de la formulación de proyectos que se justifiquen única y exclusivamente por el activismo, y que hagan una lectura más amplia del contexto, para identificar las verdaderas potencialidades del mismo a través de la solución de los problemas reales a los cuales están sometidos. Es importante, crear la conciencia de lo que implica la celebración del Día del Agua, por ejemplo, a través de mecanismos de ahorro y uso eficiente del líquido, de la importancia de la conservación del recurso como fuente que se agota, como identificación de su estado en los ámbitos local, regional y global, para hacer de él un uso sostenible que les garantice a las generaciones venideras el uso propio para desarrollar sus actividades cotidianas, en calidad, cantidad y disponibilidad.

Sin embargo, vale la pena mencionar también que, la educación ciudadana no puede depender únicamente de los medios de comunicación de masas. La escuela tiene ante sí el cometido de aportar un distanciamiento crítico (Romano, 2003) y un acercamiento eficaz.

\section{Referencias bibliográficas}

- Aguaded, J. I. (2012). La educomunicación: una apuesta de mañana, necesaria para hoy. Aularia: Revista Digital de Comunicación, 1(2), 259-261

- Badillo Mendoza, M. E. (2011). Estrategia de comunicación y educación mediada por TIC para el fomento del desarrollo sostenible en cinco colegios de Palmira. Entramado, 7(1), 128-145

- Buendía Eisman, L., Colás Bravo, M. P. \& Hernández Pina, F. (1997). Métodos de investigación en psicopedagogía. Madrid: McGraw-Hill.

- Carrasco, M. T. (1998). La educación ambiental: una estrategia flexible, un proceso y unos propósitos en permanente construcción. La experiencia de Colombia. Revista Iberoamericana de Educación, (16), 23-48.

- Del Rincón, D. A., Arnal, J., Latorre, A. y Sanz, A. (1995). Técnicas de investigación en Ciencias Sociales. Madrid: Dykinson.

- Educación, M. (2002). Política Nacional de Educación Ambiental. Bogotá D. C., República de Colombia.

- Romano García, V. (2003). Educación ciudadana y medios de comunicación. Revista de Educación, N. ${ }^{\circ}$ extra 1, 391-401 
- Sauvé, L. (2003). Perspectivas curriculares para la formación de formadores en educación ambiental. I Foro Nacional sobre la Incorporación de la Perspectiva, (13). Luis de Potosí (México).

- Soares, I. de O. (2000). Educomunicación: comunicación y tecnologías de la información en la reforma de la enseñanza americana. Diálogos de la Comunicación, 59-60, 37-152

- Wilches-Chaux, G. (2006). Brújula, bastón y lámpara para trasegar los caminos de la educación ambiental. Bogotá D. C., República de Colombia: Ministerio de Medio Ambiente, Vivienda y Desarrollo Territorial. 